PROCEEDINGS OF THE

AMERICAN MATHEMATICAL SOCIETY

Volume 130, Number 4, Pages 1103-1111

S 0002-9939(01)06140-8

Article electronically published on October 1, 2001

\title{
EXTINCTION AND DECAY ESTIMATES FOR VISCOUS HAMILTON-JACOBI EQUATIONS IN $\mathbb{R}^{N}$
}

\author{
SAID BENACHOUR, PHILIPPE LAURENÇOT, AND DIDIER SCHMITT
}

(Communicated by David S. Tartakoff)

\begin{abstract}
We consider non-negative and integrable classical solutions to the Cauchy problem $u_{t}-\Delta u+|\nabla u|^{p}=0$ when $p \in(0,+\infty)$. For $p \in(0, N /(N+1))$ we prove that any such solution vanishes identically after a finite time. For higher values of $p$ temporal decay estimates are obtained.
\end{abstract}

\section{INTRODUCTION AND MAIN RESULTS}

We consider the Cauchy problem

$$
\begin{aligned}
u_{t}-\Delta u+|\nabla u|^{p} & =0 \text { in }(0,+\infty) \times \mathbb{R}^{N}, \\
u(0) & =u_{0} \text { in } \mathbb{R}^{N},
\end{aligned}
$$

where $p \in(0,+\infty)$ and $u_{0}$ is a non-negative function in $\mathcal{B C}\left(\mathbb{R}^{N}\right) \cap L^{1}\left(\mathbb{R}^{N}\right)$. Here $\mathcal{B C}\left(\mathbb{R}^{N}\right)$ denotes the space of bounded and continuous functions in $\mathbb{R}^{N}$. For such initial data existence and uniqueness of non-negative classical solutions to (1)-(2) have been obtained by Gilding, Guedda \& Kersner 11. Within the framework of non-negative solutions the term $|\nabla u|^{p}$ in (1) acts as an absorption term and the smaller the exponent $p$ is, the stronger the absorption. The aim of this work is then to investigate some qualitative properties of non-negative solutions to (12)-(2) according to the values of $p$. More precisely we prove the noteworthy fact that any non-negative solution to (1)-(2) with initial data in $\mathcal{B C}\left(\mathbb{R}^{N}\right) \cap L^{1}\left(\mathbb{R}^{N}\right)$ vanishes identically after a finite time when $p \in(0, N /(N+1))$, a property also called extinction in finite time. Our result reads as follows.

Theorem 1. Let $u_{0}$ be a non-negative function in $\mathcal{B C}\left(\mathbb{R}^{N}\right) \cap L^{1}\left(\mathbb{R}^{N}\right)$ and denote by $u$ the unique non-negative classical solution to (11) -(2). If $p \in(0, N /(N+1))$ the solution $u$ vanishes identically after some finite time; that is, there exists $T_{\star} \in$ $[0,+\infty)$ such that

$$
u(t, x)=0 \text { for }(t, x) \in\left[T_{\star},+\infty\right) \times \mathbb{R}^{N} .
$$

It is well-known that the property of extinction in finite time is enjoyed by bounded non-negative solutions to nonlinear parabolic equations with absorption such as

$$
v_{t}-\Delta v+v^{p}=0 \text { in }(0,+\infty) \times \mathbb{R}^{N},
$$

Received by the editors March 23, 2000 and, in revised form, October 12, 2000

1991 Mathematics Subject Classification. Primary 35B40, 35B05, 35K55.

Key words and phrases. Extinction in finite time, temporal decay estimates, viscous HamiltonJacobi equations. 
when $p \in(0,1)$ (see [13, 8, 12, 10] and the references therein). For both equations (11) and (3) there is a competition between the diffusion term and the absorption term. Furthermore, for $p$ sufficiently small, the absorption is strong enough to drive any bounded non-negative solution to zero in finite time. It is worth mentioning at this point that the fact that bounded non-negative solutions to (3) vanish identically after a finite time for $p \in(0,1)$ follows easily by comparison with the corresponding ordinary differential equation. Such a device does not seem to be available for (1) and the proof of Theorem 1 requires a different approach. We also point out that the exponent below which the extinction in finite time is guaranteed does depend on the space dimension $N$ for (1) and that we require the integrability of the solution to (11) in addition to its boundedness for extinction in finite time to occur, both facts being in contrast with (3). A natural question is then whether solutions to (1) decaying faster for large values of $|x|$ could exhibit this extinction phenomenon for $p \in[N /(N+1), 1)$. In this connection it has been pointed out to us by Professor Brian Gilding that, if $u_{0} \in \mathcal{B C}\left(\mathbb{R}^{N}\right)$ is non-negative and compactly supported, the corresponding solution to (1)-(2) vanishes identically after a finite time for every $p \in(0,1)$. This follows from [11, Theorem 19]. Further results in that direction are still in progress.

Remark 1. The proof of Theorem 1 relies on a precise study of the behaviour of global quantities involving $u$ such as $L^{q}$-norms, and does not provide information about the way extinction takes place. Such information is already available for (3) when $N=1$ (see, e.g., [12] and the references therein).

For higher values of $p$ we establish some temporal decay estimates for the $L^{\infty}$ norm of non-negative and integrable solutions to (1I). More precisely our result reads:

Theorem 2. Let $u_{0}$ be a non-negative function in $\mathcal{B C}\left(\mathbb{R}^{N}\right) \cap L^{1}\left(\mathbb{R}^{N}\right)$ and denote by $u$ the unique non-negative classical solution to (1)-(2). There is a constant $\kappa$ depending only on $p, N,\left\|u_{0}\right\|_{L^{1}}$ and $\left\|u_{0}\right\|_{L^{\infty}}$ such that:

(i) if $p=N /(N+1)$, then

$$
\|u(t)\|_{L^{\infty}} \leq \kappa \exp (-\kappa t), \quad t \in(0,+\infty) ;
$$

(ii) if $p>N /(N+1)$, then

$$
\|u(t)\|_{L^{\infty}} \leq \kappa t^{-\beta_{p, N}}, \quad t \in(0,+\infty)
$$

$$
\beta_{p, N}=\left\{\begin{array}{lll}
N /(p(N+1)-N) & \text { if } & p \in(N /(N+1),(N+2) /(N+1)), \\
N / 2 & \text { if } & p \geq(N+2) /(N+1) .
\end{array}\right.
$$

Remark 2. The constant $\kappa$ in Theorem 2 actually only depends on $p, N$ and $\left\|u_{0}\right\|_{L^{1}}$ if $p>N /(N+1)$.

Observe that, for $p \in[N /(N+1),(N+2) /(N+1))$, the temporal decay estimates (4) and (5) are faster for large times than the one which follows from the heat equation by comparison. Therefore the absorption term is dominating in this case. The situation is reversed for $p \geq(N+2) /(N+1)$ where the diffusion term rules out the absorption at large times. Notice also that the exponent $\beta_{p, N}$ varies continuously in $[N / 2,+\infty)$ as $p \in(N /(N+1),(N+2) /(N+1)]$.

It follows from (5]) that, under the assumptions of Theorem[2] the $L^{\infty}$-norm of $u$ decays at least as $t^{-\beta_{p, N}}$. However, there might be initial data for which $\|u(t)\|_{L^{\infty}}$ 
decays more rapidly. Still, this is not the case for $p>(N+2) /(N+1)$, as follows from a more precise study of the large time behaviour of $u[4]$. The situation is completely different for $p=1$ where exponential or arbitrarily large polynomial decay estimates of $\|u(t)\|_{L^{\infty}}$ are available for initial data decaying sufficiently rapidly for large values of $|x|$ (see [5] and the references therein). For intermediate values of $p \in$ $(1,(N+2) /(N+1)]$ it follows from [1. Corollary 3.5] that $\|u(t)\|_{L^{\infty}}$ cannot decay arbitrarily fast. We state below a slight improvement of this result (case (b) below), the proof relying partly on different arguments.

Proposition 3. Let $u_{0}$ be a non-negative function in $\mathcal{B C}\left(\mathbb{R}^{N}\right) \cap L^{1}\left(\mathbb{R}^{N}\right)$ and denote by $u$ the unique non-negative classical solution to (12)-(2). Suppose further that

(a) either $p \in(1,(N+2) /(N+1)]$ and there is $\alpha>\alpha_{p, N}:=(2-p) /$ $(2(p-1))-(N / 2)$ such that

$$
\|u(t)\|_{L^{\infty}} \leq C t^{-(N / 2)-\alpha} \text { for } t \geq 1,
$$

(b) or $p=(N+2) /(N+1)$ and there is $\gamma>N+1$ such that

$$
\|u(t)\|_{L^{\infty}} \leq C t^{-N / 2}(\ln t)^{-\gamma} \text { for } t \geq 1 .
$$

Then $u \equiv 0$.

For $p \in(1,(N+2) /(N+1))$ it follows from Theorem 2 and Proposition 3 that the exponent of possible polynomial decay rates of $\|u(t)\|_{L^{\infty}}$ ranges in $\left[\beta_{p, N}, \alpha_{p, N}+\right.$ $N / 2$. The upper bound $\alpha_{p, N}+N / 2$ is optimal as there is at least one solution to (1) with $L^{\infty}$-norm decaying exactly as $t^{-\alpha_{p, N}-(N / 2)}[3]$. If $p=(N+2) /(N+1)$, we have $\alpha_{p, N}=0$ and $\beta_{p, N}=N / 2$; consequently $t^{-N / 2}$ is the optimal polynomial decay rate for $\|u(t)\|_{L^{\infty}}$ in that case. Notice however that, in view of the results already known for (3) (see [9] and the references therein), it is expected that $\|u(t)\|_{L^{\infty}}$ could decay exactly as $t^{-N / 2}(\ln t)^{-(N+1)}$ for some initial data, this decay rate being the largest of this kind allowed by Proposition 3 .

The proofs of Theorems 1 and 2 are actually split into three parts: we first consider the case $p \in(0,1)$ and establish a differential inequality for a suitable auxiliary function. Exploiting this inequality yields Theorem 1 and Theorem 2 for $p \in[N /(N+1), 1)$. Theorem 2 for $p=1$ follows next from a Sobolev inequality by a Moser technique borrowed from [7, Section 4]. The proof of Theorem 2] for $p>1$ relies on gradient estimates derived in [2] Theorem 1]. We finally end up the paper with a sketch of the proof of Proposition 3.

\section{The CASE $p \in(0,1)$}

In this section we assume that $p \in(0,1)$ and we consider a non-negative function $u_{0}$ in $\mathcal{B C}\left(\mathbb{R}^{N}\right) \cap L^{1}\left(\mathbb{R}^{N}\right), u_{0} \not \equiv 0$. By [11, Theorems $2,4,5$ and 7 ] there is a unique classical solution

$$
u \in \mathcal{C}\left([0,+\infty) \times \mathbb{R}^{N}\right) \cap \mathcal{C}^{1,2}\left((0,+\infty) \times \mathbb{R}^{N}\right)
$$

to (1)-(2) enjoying the following properties:

$$
\begin{aligned}
& t \mapsto\|u(t)\|_{L^{\infty}} \text { is a non-increasing function, } \\
& t \mapsto\|\nabla u(t)\|_{L^{\infty}} \text { is a non-increasing function, } \\
& \|\nabla u(t)\|_{L^{\infty}} \leq C_{0}(t-s)^{-1 / p}\|u(s)\|_{L^{\infty}}^{1 / p}, \quad 0 \leq s<t,
\end{aligned}
$$


where $C_{0}$ is a positive constant depending only on $p$ and $N$. Furthermore it is easy to check by integration of (1) over $\mathbb{R}^{N}$ that $u \in L^{\infty}\left(0,+\infty ; L^{1}\left(\mathbb{R}^{N}\right)\right)$ and

$$
t \mapsto\|u(t)\|_{L^{1}} \text { is a non-increasing function. }
$$

We next fix $\vartheta>((2 p-N) / N)+$ and put

$$
a=N+\vartheta(N+1), \quad b=N(\vartheta+1)-p .
$$

Notice that, as $p \in(0,1)$ and $\vartheta>((2 p-N) / N)_{+}$, we have

$$
b>p .
$$

The main step of our analysis of the case $p \in(0,1)$ is the following lemma.

Lemma 4. The function $t \mapsto(1+t)\|u(t)\|_{L^{\infty}}^{a}$ belongs to $L^{1}(0,+\infty)$ and the auxiliary function $y$ defined by

$$
y(t)=\int_{t}^{\infty}(s-t)\|u(s)\|_{L^{\infty}}^{a} d s, \quad t \in[0,+\infty),
$$

belongs to $W^{2, \infty}(0,+\infty)$ and satisfies the differential inequality

$$
y^{\prime}(t)+C_{1} y(t)^{1+\alpha} \leq 0, \quad t \in[0,+\infty),
$$

for some constant $C_{1}$ depending only on $N, p, \vartheta$ and $\left\|u_{0}\right\|_{L^{1}}$, and

$$
\alpha=\frac{p(N+1)-N}{(b-p)(N+1)+N}>-1 .
$$

Proof of Lemma 4. In the following we denote by $C$ any positive constant depending only on $N, p, \vartheta$ and $\left\|u_{0}\right\|_{L^{1}}$. We put

$$
t_{\star}=\inf \{t>0, \quad u(t) \equiv 0\},
$$

and notice that $t_{\star}>0$ as $u_{0} \not \equiv 0$. Also, as $u(t)$ belongs to $L^{1}\left(\mathbb{R}^{N}\right)$, an equivalent definition of $t_{\star}$ is

$$
t_{\star}=\inf \{t>0, \quad|\nabla u(t)| \equiv 0\} .
$$

Consider $T \in\left(0, t_{\star}\right)$ and $t \in(0, T)$. We integrate (1) over $(t, T) \times \mathbb{R}^{N}$ and use the non-negativity of $u$ and (9) to obtain

$$
\int_{t}^{T} \int|\nabla u(s, x)|^{p} d x d s \leq\left\|u_{0}\right\|_{L^{1}} .
$$

As $p \in(0,1)$ we are not able to use directly a Sobolev inequality. We thus proceed as follows. Recalling that $b=N(\vartheta+1)-p>0$ the above inequality yields

$$
\int_{t}^{T}\|\nabla u(s)\|_{L^{\infty}}^{-b}\|\nabla u(s)\|_{L^{N(1+\vartheta)}}^{N(1+\vartheta)} d s \leq C .
$$

On the one hand the Gagliardo-Nirenberg inequality [14] Theorem II.2.2] and (9) ensure that

$$
\|u(s)\|_{L^{\infty}} \leq C\left\|u_{0}\right\|_{L^{1}}^{\vartheta / a}\|\nabla u(s)\|_{L^{N(1+\vartheta)}}^{N(1+\vartheta) / a}, \quad s \in(t, T) .
$$

On the other hand it follows from (8) that

$$
\|\nabla u(s)\|_{L^{\infty}}^{-b} \geq C_{0}^{-b}(s-t)^{b / p}\|u(t)\|_{L^{\infty}}^{-b / p}, \quad s \in(t, T) .
$$

Combining (12)-(14) then yields

$$
\int_{t}^{T}(s-t)^{b / p}\|u(s)\|_{L^{\infty}}^{a} d s \leq C\|u(t)\|_{L^{\infty}}^{b / p}, \quad t \in(0, T) .
$$


Letting $T \rightarrow t_{\star}$ we conclude that

$$
\int_{t}^{t_{\star}}(s-t)^{b / p}\|u(s)\|_{L^{\infty}}^{a} d s \leq C\|u(t)\|_{L^{\infty}}^{b / p}, \quad t \in\left(0, t_{\star}\right) .
$$

A first consequence of (15) is that

$$
t \mapsto(1+t)\|u(t)\|_{L^{\infty}}^{a} \in L^{1}(0,+\infty) .
$$

Indeed, if $\delta$ is a fixed real number in $\left(0, t_{\star}\right)$, we have by (6) and (10)

$$
\begin{aligned}
& \int_{0}^{\infty}(1+s)\|u(s)\|_{L^{\infty}}^{a} d s \\
& \leq(1+\delta) \quad\left(\delta\left\|u_{0}\right\|_{L^{\infty}}^{a}+\delta^{-b / p} \int_{\delta}^{t_{\star}} s^{b / p}\|u(s)\|_{L^{\infty}}^{a} d s\right) \\
& \leq(1+\delta) \quad\left(\delta\left\|u_{0}\right\|_{L^{\infty}}^{a}+\delta^{-b / p} \int_{0}^{t_{\star}} s^{b / p}\|u(s)\|_{L^{\infty}}^{a} d s\right) .
\end{aligned}
$$

Now the right-hand side of the above inequality is finite by (15), hence (16). Consequently the function $y$ defined in Lemma 4 is well-defined on $[0,+\infty)$ and belongs to $W^{2, \infty}(0,+\infty)$ by (16) and (6) with

$$
y^{\prime}(t)=-\int_{t}^{\infty}\|u(s)\|_{L^{\infty}}^{a} d s \text { and } y^{\prime \prime}(t)=\|u(t)\|_{L^{\infty}}^{a}, \quad t \in[0,+\infty) .
$$

In addition, as $u_{0} \not \equiv 0, y(0)>0$.

Another consequence of (15) is a differential inequality for $y$. Indeed recalling (10) and (16) we infer from the Jensen inequality that, for $t \in\left(0, t_{\star}\right)$,

$$
y(t)^{b / p}\left(\int_{t}^{t_{\star}}\|u(s)\|_{L^{\infty}}^{a} d s\right)^{1-b / p} \leq \int_{t}^{t_{\star}}(s-t)^{b / p}\|u(s)\|_{L^{\infty}}^{a} d s,
$$

which yields, together with (15),

$$
y(t)^{b / p}\left(-y^{\prime}(t)\right)^{1-b / p} \leq C y^{\prime \prime}(t)^{b /(a p)}, \quad t \in\left(0, t_{\star}\right) .
$$

Consequently $y$ satisfies the following differential inequality:

$$
y(t)^{a} \leq C y^{\prime \prime}(t)\left(-y^{\prime}(t)\right)^{(1-p / b) a}, \quad t \in\left(0, t_{\star}\right) .
$$

We multiply the above inequality by $\left(-y^{\prime}(t)\right)$ and integrate over $\left(t, t_{\star}\right)$ to obtain, since $y^{\prime}(t) \leq 0$,

$$
y(t)^{1+a} \leq C\left(-y^{\prime}(t)\right)^{2+(1-p / b) a}, \quad t \in\left(0, t_{\star}\right) .
$$

As $2+(1-p / b) a=(1+a) /(1+\alpha)$ and $\alpha>-1$ by (10), the inequality (11) follows at once from the above inequality for $t \in\left[0, t_{\star}\right)$. The extension of (11) to $\left[t_{\star},+\infty\right)$ (if $t_{\star}<+\infty$ ) is then straightforward as $y$ identically vanishes on $\left[t_{\star},+\infty\right.$ ).

We are now in a position to prove Theorems 1 and 2 for $p \in[N /(N+1), 1)$. First observe that $\alpha=0$ if $p=N /(N+1)$ while $\alpha<0$ if $p \in(0, N /(N+1))$ and $\alpha>0$ if $p \in(N /(N+1), 1)$. We thus distinguish three cases below.

Proof of Theorem [1. As $p \in(0, N /(N+1))$ we have $1+\alpha \in(0,1)$. Consequently we infer from Lemma 4 that $y(t)=0$ for $t \geq T_{e}$ where

$$
T_{e}=\frac{y(0)^{-\alpha}}{|\alpha| C_{1}}<+\infty \text {. }
$$


We thus conclude that $\|u(t)\|_{L^{\infty}}^{a}=y^{\prime \prime}(t)=0$ for $t \geq T_{e}$ and the proof of Theorem 1 is complete.

Proof of Theorem $0($ for $p \in[N /(N+1), 1))$. We first consider the case $p=$ $N /(N+1)$. Then $\alpha=0$ and it follows from Lemma 4 that

$$
y(t) \leq y(0) \exp \left(-C_{1} t\right), \quad t \in[0,+\infty) .
$$

Let $t \in(0,+\infty)$. By ([6) we have

$$
\frac{t^{2}}{8}\|u(t)\|_{L^{\infty}}^{a} \leq \int_{t / 2}^{t}\left(s-\frac{t}{2}\right)\|u(s)\|_{L^{\infty}}^{a} d s \leq y\left(\frac{t}{2}\right) .
$$

Combining the above two inequalities yields (44) for $t \in[1,+\infty)$ and a further application of (6) entails (4) for $t \in[0,1]$.

We next turn to the case $p \in(N /(N+1), 1)$. Then $1+\alpha>1$ and it follows from Lemma 4 that

$$
y(t) \leq\left(\alpha C_{1} t\right)^{-1 / \alpha}, \quad t \in(0,+\infty)
$$

We then proceed as above to conclude that (5) holds true for $p \in(N /(N+1), 1)$.

Recalling (8) temporal decay estimates for $\|\nabla u\|_{L^{\infty}}$ follow from Theorem 2 for $p \in[N /(N+1), 1)$.

Corollary 5. Let $u_{0}$ be a non-negative function in $\mathcal{B C}\left(\mathbb{R}^{N}\right) \cap L^{1}\left(\mathbb{R}^{N}\right)$ and denote by $u$ the unique non-negative classical solution to (11)-(2). There is a constant $\kappa_{1}$ depending only on $p, N,\left\|u_{0}\right\|_{L^{1}}$ and $\left\|u_{0}\right\|_{L^{\infty}}$ such that:

(i) if $p=N /(N+1)$, then

$$
\|\nabla u(t)\|_{L^{\infty}} \leq \kappa_{1} \exp \left(-\kappa_{1} t\right), \quad t \in(0,+\infty)
$$

(ii) if $p \in(N /(N+1), 1)$, then

$$
\|\nabla u(t)\|_{L^{\infty}} \leq \kappa_{1} t^{-(N+1) /(p(N+1)-N)}, \quad t \in(0,+\infty) .
$$

As in Theorem 2 (see Remark 21) the constant $\kappa_{1}$ in Corollary 5 only depends on $p, N$ and $\left\|u_{0}\right\|_{L^{1}}$ if $p \in(N /(N+1), 1)$.

\section{The CASE $p=1$}

Let $u_{0}$ be a non-negative function in $\mathcal{B C}\left(\mathbb{R}^{N}\right) \cap L^{1}\left(\mathbb{R}^{N}\right)$. By [1] there is a unique classical solution $u$ to (17)-(2) satisfying ([6)-(17). In the following we denote by $C$ any positive constant depending only on $N$ and $\left\|u_{0}\right\|_{L^{1}}$.

In order to prove Theorem 2 for $p=1$ we employ a Moser technique as in 7 . Section 4]. Consider $r \geq 1, s_{2} \in(0,+\infty)$ and $s_{1} \in\left[0, s_{2}\right)$. It follows from (11) after multiplication by $r u^{r-1}$ and integration over $\left(s_{1}, s_{2}\right) \times \mathbb{R}^{N}$ that

$$
\left\|u\left(s_{2}\right)\right\|_{L^{r}}^{r}+\int_{s_{1}}^{s_{2}} \int\left|\nabla\left(u^{r}\right)(s, x)\right| d x d s \leq\left\|u\left(s_{1}\right)\right\|_{L^{r}}^{r}
$$

We next use the Sobolev inequality [6] Théorèmes VIII.7 \& IX.9] to conclude that

$$
\int_{s_{1}}^{s_{2}}\left\|u^{r}(\sigma)\right\|_{L^{1^{*}}} d \sigma \leq C\left\|u\left(s_{1}\right)\right\|_{L^{r}}^{r}
$$

where $1^{*}=\infty$ if $N=1$ and $1^{*}=N /(N-1)$ otherwise.

Fix $t \in(0,+\infty)$. If $N=1$, we choose $r=1, s_{1}=0$ and $s_{2}=t$ in (17) and use (6) to obtain (5) for $p=1$. Assume now that $N \geq 2$. As $u$ is non-negative, 
$s \mapsto\|u(s)\|_{L^{q}}$ is a non-increasing function for each $q \in[1, \infty]$ and we infer from (17) that

$$
\left\|u\left(s_{2}\right)\right\|_{L^{r N /(N-1)}} \leq C^{1 / r}\left(s_{2}-s_{1}\right)^{-1 / r}\left\|u\left(s_{1}\right)\right\|_{L^{r}}, \quad 0 \leq s_{1}<s_{2} .
$$

Introducing for $k \geq 0$ the sequences $r_{k}=(N /(N-1))^{k}$ and $t_{k}=t\left(1-2^{-(k+1)}\right)$, we proceed as in 7, Section 4] and write (18) with $r=r_{k}, s_{1}=t_{k}$ and $s_{2}=t_{k+1}$. Arguing by induction we eventually arrive at the following inequality:

$$
\|u(t)\|_{L^{r_{k}}} \leq\left\|u\left(t_{k}\right)\right\|_{L^{r_{k}}} \leq C^{\alpha_{k}} 2^{\beta_{k}} t^{-\alpha_{k}}\|u(t / 2)\|_{L^{1}}
$$

for $k \geq 1$ with

$$
\alpha_{k}=\sum_{n=0}^{k-1}\left(\frac{N-1}{N}\right)^{n} \text { and } \beta_{k}=\sum_{n=0}^{k-1}(n+2)\left(\frac{N-1}{N}\right)^{n}, \quad k \geq 0 .
$$

We may now let $k \rightarrow+\infty$ in (19) to obtain (5) for $p=1$.

\section{The CASE $p>1$}

Let $u_{0}$ be a non-negative function in $\mathcal{B C}\left(\mathbb{R}^{N}\right) \cap L^{1}\left(\mathbb{R}^{N}\right)$. By [1] there is a unique classical solution $u$ to (11)-(2) satisfying (6) -(7) and the non-negativity of $u$ and (1) entails that (9) also holds true. We moreover infer from [2, Theorem 1] that there is a constant $\gamma_{p}$ depending only on $p$ such that

$$
|\nabla u(t, x)| \leq \gamma_{p} t^{-1 / p} u(t, x)^{1 / p}, \quad(t, x) \in(0,+\infty) \times \mathbb{R}^{N} .
$$

In the following we denote by $C$ any positive constant depending only on $p, N$ and $\left\|u_{0}\right\|_{L^{1}}$. Let $t \in(0,+\infty)$. By (9) and the Gagliardo-Nirenberg inequality [14, Theorem II.2.2] we have

$$
\|u(t)\|_{L^{\infty}} \leq C\|\nabla u(t)\|_{L^{\infty}}^{N /(N+1)}\left\|u_{0}\right\|_{L^{1}}^{1 /(N+1)} .
$$

Combining the above two estimates yields

$$
\|u(t)\|_{L^{\infty}} \leq C t^{-N /(p(N+1)-N)}, \quad t \in(0,+\infty),
$$

hence (5) for $p \in(1,(N+2) /(N+1))$.

Observe finally that $u$ is a subsolution to the linear heat equation from which we deduce that

$$
\|u(t)\|_{L^{\infty}} \leq C t^{-N / 2}, \quad t \in(0,+\infty) .
$$

Now it is clear that the decay estimate (22) is faster than the decay estimate (21) for large times when $p \geq(N+2) /(N+1)$, hence (5) and the proof of Theorem 2 is complete.

As a consequence of Theorem 2, (201) and [2, estimate (6), p. 2001] we obtain temporal decay estimates for $\|\nabla u\|_{L^{\infty}}$.

Corollary 6. Let $u_{0}$ be a non-negative function in $\mathcal{B C}\left(\mathbb{R}^{N}\right) \cap L^{1}\left(\mathbb{R}^{N}\right)$ and denote by $u$ the unique non-negative classical solution to (11)-(21). There is a constant $\kappa_{1}$ depending only on $p, N$ and $\left\|u_{0}\right\|_{L^{1}}$ such that, if $p>1$, then

$$
\|\nabla u(t)\|_{L^{\infty}} \leq \kappa_{1} t^{-\left((N+1) \beta_{p, N}\right) / N}, \quad t \in(0,+\infty) .
$$




\section{A sketch of the proof of Proposition 3}

It follows from (1) that, for $t \geq s \geq 2$,

$$
\|u(s)\|_{L^{1}}-\|u(t)\|_{L^{1}} \leq C(p) \int_{s}^{t}\left\|\nabla\left(u^{(p-1) / p}\right)(\sigma)\right\|_{L^{\infty}}^{p}\|u(\sigma)\|_{L^{1}} d \sigma .
$$

We infer from [2, Eq. (17)] and (9) that

$$
\|u(s)\|_{L^{1}}-\|u(t)\|_{L^{1}} \leq C(p)\|u(s)\|_{L^{1}} \int_{s / 2}^{t}\|u(\sigma)\|_{L^{\infty}}^{p-1} \sigma^{-p / 2} d \sigma .
$$

But it is easy to check that the assumptions $(a)$ or $(b)$ imply $\sigma \mapsto\|u(\sigma)\|_{L^{\infty}}^{p-1} \sigma^{-p / 2}$ belongs to $L^{1}(1,+\infty)$. Consequently we may find $s_{0}$ large enough such that

$$
\left\|u\left(s_{0}\right)\right\|_{L^{1}}-\|u(t)\|_{L^{1}} \leq \frac{1}{2}\left\|u\left(s_{0}\right)\right\|_{L^{1}} \quad \text { for } t \geq s_{0} .
$$

We argue as in the proof of [1, Corollary 3.5] to conclude that $u \equiv 0$.

\section{ACKNOWLEDGMENTS}

We thank Professor Brian Gilding and the referee for their valuable remarks on the first version of this paper.

\section{REFERENCES}

1. M. Ben-Artzi and H. Koch, Decay of mass for a semilinear parabolic equation, Comm. Partial Differential Equations 24 (1999), 869-881. MR 2000a:35098

2. S. Benachour and $\mathrm{Ph}$. Laurençot, Global solutions to viscous Hamilton-Jacobi equations with irregular initial data, Comm. Partial Differential Equations 24 (1999), 1999-2021. MR 2000g:35091

3. S. Benachour and $\mathrm{Ph}$. Laurençot, Very singular solutions to a nonlinear parabolic equation with absorption. I. Existence, Proc. Roy. Soc. Edinburgh Sect. A, 131 (2001), 27-44. CMP 2001:09

4. S. Benachour, Ph. Laurençot and D. Schmitt, in preparation.

5. S. Benachour, B. Roynette and P. Vallois, Asymptotic estimates of solutions of $u_{t}-\Delta u=$ $-|\nabla u|$ in $\mathbb{R}_{+} \times \mathbb{R}^{d}, d \geq 2$, J. Funct. Anal. 144 (1997), 301-324. MR 97m:35118

6. H. Brézis, Analyse fonctionnelle. Théorie et applications, Masson, Paris, 1983. MR 85a:46001

7. L.C. Evans, Regularity properties for the heat equation subject to nonlinear boundary constraints, Nonlinear Anal. 1 (1977), 593-602. MR 58:29276

8. L.C. Evans and B.F. Knerr, Instantaneous shrinking of the support of nonnegative solutions to certain nonlinear parabolic equations and variational inequalities, Illinois J. Math. 23 (1979), 153-166. MR 80d:35082

9. V.A. Galaktionov and J.L. Vazquez, Asymptotic behaviour of nonlinear parabolic equations with critical exponents. A dynamical systems approach, J. Funct. Anal. 100 (1991), 435-462. MR 92k:35128

10. V.A. Galaktionov and J.L. Vazquez, Continuation of blowup solutions of nonlinear heat equations in several space dimensions, Comm. Pure Appl. Math. 50 (1997), 1-67. MR 97h:35085

11. B.H. Gilding, M. Guedda and R. Kersner, The Cauchy problem for $u_{t}=\Delta u+|\nabla u|^{q}$, prépublication LAMFA 28, Université de Picardie, 1998.

12. M.A. Herrero and J.J.L. Velázquez, Approaching an extinction point in one-dimensional semilinear heat equations with strong absorption, J. Math. Anal. Appl. 170 (1992), 353-381. MR 93k:35043 
13. A.S. Kalashnikov, The propagations of disturbances in problems of non-linear heat conduction with absorption, Comput. Math. Math. Phys. 14 (1974), 70-85.

14. O.A. Ladyženskaja, V.A. Solonnikov and N.N. Ural'ceva, Linear and quasilinear equations of parabolic type, Transl. Math. Monogr. 23, Amer. Math. Soc., Providence, 1968. MR 39:3159b

Institut Elie Cartan - Nancy, Université de Nancy I, BP 239, F-54506 Vandeuvre Les NANCY CEDEX, FRANCE

E-mail address: benachou@iecn.u-nancy.fr

Institut Elie Cartan - Nancy, Université de Nancy I, BP 239, F-54506 Vandeuvre les NANCY CEDEX, France

E-mail address: laurenco@iecn.u-nancy.fr

Current address: Mathématiques pour l'Industrie et la Physique, UNR CNRS 5640, Université

Paul Sabatier-Toulouse 3, 118, route de Narbonne, F-31062 Toulouse Cedex 4, France

E-mail address: laurencot@mip.ups-tlse.fr

Institut Elie Cartan - Nancy, Université de NAncy I, BP 239, F-54506 Vandeuvre les NANCY CEDEX, France

E-mail address: dschmitt@iecn.u-nancy.fr 